\title{
Maheshvara regulates JAK/STAT signaling by interacting and stabilizing hopscotch transcripts which leads to apoptosis in Drosophila melanogaster
}

\author{
Bhawana Maurya ${ }^{1}$, Satya Surabhi ${ }^{1,2}$, Rituparna Das ${ }^{1}$, Pranjali Pandey ${ }^{1}$, Ashim Mukherjee ${ }^{1}$ and Mousumi Mutsuddi id
}

\begin{abstract}
Maheshvara (mahe), an RNA helicase that is widely conserved across taxa, regulates Notch signaling and neuronal development in Drosophila. In order to identify novel components regulated by mahe, transcriptome profiling of ectopic mahe was carried out and this revealed striking upregulation of JAK/STAT pathway components like upd1, upd2, upd3, and socs36E. Further, significant downregulation of the pathway components in mahe loss-of-function mutant as well as upon lowering the level of mahe by RNAi, supported and strengthened our transcriptome data. Parallelly, we observed that mahe, induced caspase-dependent apoptosis in photoreceptor neurons, and this phenotype was significantly modulated by JAK/STAT pathway components. RNA immunoprecipitation unveiled the presence of JAK/STAT tyrosine kinase hopscotch (hop) transcripts in the complex immunoprecipitated with Mahe, which ultimately resulted in stabilization and elevation of hop transcripts. Additionally, we also observed the surge in activity of downstream transcription factor Stat92E, which is indicative of activation of the JAK/STAT signaling, and this in turn led to apoptosis via upregulation of hid. Taken together, our data provide a novel regulation of JAK/STAT pathway by RNA helicase Maheshvara, which ultimately promotes apoptosis.
\end{abstract}

\section{Introduction}

With the development of a deeper understanding of the importance of RNA in maintaining the fidelity of biological processes, the pivotal roles played by RNA binding proteins $(\mathrm{RBPs})^{1,2}$ such as the DEAD box RNA helicases (DBRHs) are being unraveled. The DBRHs are a set of multitasking proteins that are involved in multitudes of RNA processes depending largely on the partners they interact with in various biological contexts ${ }^{3,4}$. The involvement of DBRHs in RNA processing events in neurons implicates their role in numerous neurological disorders ${ }^{4,5}$. High-throughput

Correspondence: Mousumi Mutsuddi (mousumi@bhu.ac.in)

'Department of Molecular and Human Genetics, Institute of Science, Banaras Hindu University, Varanasi 221005 Uttar Pradesh, India. ${ }^{2}$ Present address:

Department of Molecular and Developmental Biology, Albert Einstein College of Medicine, New York, NY 10461, USA

Edited by K. McCall approaches have identified mutations in DEAD box helicase genes as major cause of neurodevelopmental disabilities, these include DDX59, DDX6, DHX37, DHX30, DHX16, DHX34, and DDX54 ${ }^{6-9}$.

We have previously characterized maheshvara (mahe), a Drosophila homolog of human DDX59, as a novel modulator of Notch signaling ${ }^{10}$. Misregulated mahe leads to neuronal defects and reduced lifespan in Drosophila, which parallels similarities with patients having frameshift deletion in DDX59 presenting orofaciodigital syndrome associated with broad neurological defects ${ }^{11}$.

mahe and its human orthologues have been implicated in disease and development of nervous system. To delve deeper into the mechanism of the neuronal defects caused due to ectopic mahe, we carried out whole transcriptome analysis. Interestingly, we observed significant upregulation of JAK/STAT pathway components. In addition, alike

\section{(c) The Author(s) 2021}

(c) (i) Open Access This article is licensed under a Creative Commons Attribution 4.0 International License, which permits use, sharing, adaptation, distribution and reproduction cc) in any medium or format, as long as you give appropriate credit to the original author(s) and the source, provide a link to the Creative Commons license, and indicate if changes were made. The images or other third party material in this article are included in the article's Creative Commons license, unless indicated otherwise in a credit line to the material. If material is not included in the article's Creative Commons license and your intended use is not permitted by statutory regulation or exceeds the permitted use, you will need to obtain permission directly from the copyright holder. To view a copy of this license, visit http://creativecommons.org/licenses/by/4.0/. 
to the case of many DEAD box helicase associated neurological diseases ${ }^{12,13}$, we also noted that overexpression of mahe leads to increased apoptosis in the developing photoreceptor neurons. Drosophila JAK/STAT signaling, though relatively simpler when compared to that of vertebrates, regulates patterning, organizer establishment, proliferation, apoptosis and maintains the size of the adult Drosophila eye ${ }^{14}$. Mechanistically, secreted ligand family unpaired (upd) and upd like proteins (upd2 and upd3) binding dimerizes the receptor (domeless/dome) and activates the receptor-associated JAK tyrosine kinases (hopscotch/hop). In the canonical pathway, these tyrosine kinases phosphorylate themselves and their associated receptors and this creates docking sites for signaltransducer and activator of transcription protein at $92 \mathrm{E}$ (Stat92E). Stat92E transcription factor dimerizes upon phosphorylation and enters into the nucleus to activate or repress transcription of target genes such as socs $36 E^{15,16}$. During development, the decision of a cell to die or survive requires the integration of several regulatory pathways among which JAK/STAT signaling also plays a significant role. In our study, genetic analysis of mahe with members of JAK/STAT pathway displayed modulation of apoptotic phenotype in the developing photoreceptor neurons. Our study has uncovered the novel role of an RNA helicase Mahe that binds to hop tyrosine kinase transcripts, and leads to a surge in its levels resulting in activation of JAK/STAT signaling. This ectopic JAK/STAT activation culminates into hid mediated cell death. To the best of our knowledge, this is the first report in either vertebrates or invertebrates, where the pivotal role played by an RNA helicase in activating JAK/STAT cascade has been unraveled. This activation ultimately ensues in an upregulation of hid mediated apoptosis.

\section{Results}

Transcriptome analysis of mahe reveals upregulation of JAK/STAT pathway components

For dissecting out the molecular factors regulated by mahe during development, transcriptome analysis using RNA extracted from GMR-GAL4 driven mahe overexpressed eye tissues were compared to that of the transcript levels in GMR-GAL4 controls. RNA-sequencing analysis revealed a total of 12,225 differentially regulated genes (with fold change $>0.5$ ). Out of these, 1080 genes were found to be upregulated and 519 genes were downregulated significantly (with fold change $>1.5$ ). For functional annotation of differentially expressed genes mined from our RNA-Seq data, NIH DAVID 6.8 (The Database for Annotation, Visualization, and Integrated Discovery) was used. Gene Ontology (GO) analysis, identified the biological activities and molecular pathways affected in response to mahe overexpression (Fig. 1a-d).
Interestingly, DAVID analysis revealed positive regulation of JAK/STAT cascade (Fig. 1a). To reconfirm our RNA-Seq data, real-time PCR was done in mahe loss-offunction mutant, remarkably each of the three Drosophila JAK/STAT ligands upd1, upd2, upd3, and socs36E, the downstream target of the transcription factor Stat92E were significantly downregulated (Fig. 1f). As there were very few first instar escaper larvae of mahe loss-offunction mutant and none developed up to third instar larval stage, further analysis was done using mahe-RNAi. In addition, in line with that of mahe mutants, JAK/STAT pathway components were downregulated upon lowering the levels of mahe by RNAi (Fig. 1f). Further, in agreement with the above results ectopically expressed mahe tissue revealed upregulation of the JAK/STAT signaling components, and this corresponded with the RNA-Seq data (Fig. 1e, f). Together, these data indicated mahe to be a positive regulator of JAK/STAT pathway.

\section{mahe induces Caspase-dependent cell death in a dosage sensitive manner}

Parallelly, a gain-of-function assay was carried out and the effect of ectopic expression of mahe was monitored in the developing photoreceptors. An HA tagged full length Mahe protein was ectopically expressed posterior to the morphogenetic furrow using GMR-GAL4 driver. Overexpression of mahe resulted in rough and reduced eye phenotype when compared to the GMR-GAL4 control adult eye phenotype (Fig. $2 b, b^{\prime}$ and $c, c^{\prime}$ to $a, a^{\prime}$ ). The rough eye phenotype was found to be largely due to apoptosis, as seen by acridine orange staining as well as immunostaining of cleaved caspase and the immunofluorescence signal was dosage sensitive to the levels of mahe expression (Fig. 2g, l and h, $\mathrm{m}$ to f, k). Cell survival is facilitated by factors which directly inhibit caspases. DIAP1 and P35 are such known inhibitors of caspases. Our observation was further consolidated by the significant rescue of mahe associated eye roughness and apoptosis by DIAP1 and P35 (Fig. 2d, d', i, n and e, e', j, o to $\left.b, b^{\prime}, g, l\right)$. Quantitative analysis of cleaved caspase staining in terms of intensity per unit area was indicated by prominent reduction in apoptotic cells when P35 and DIAP1 are coexpressed with mahe $(2 \mathrm{p})$. These results indicate that mahe induces cell death in canonical caspase-dependent manner and its level must be tightly regulated for proper development and differentiation of Drosophila eye.

mahe induced apoptosis is mediated via surge in JAK/STAT pathway components

Since, major components of the JAK/STAT pathway were upregulated as depicted in the RNA-Sequencing data, we next sought to identify whether the components of JAK/STAT pathway themselves alter the mahe induced 


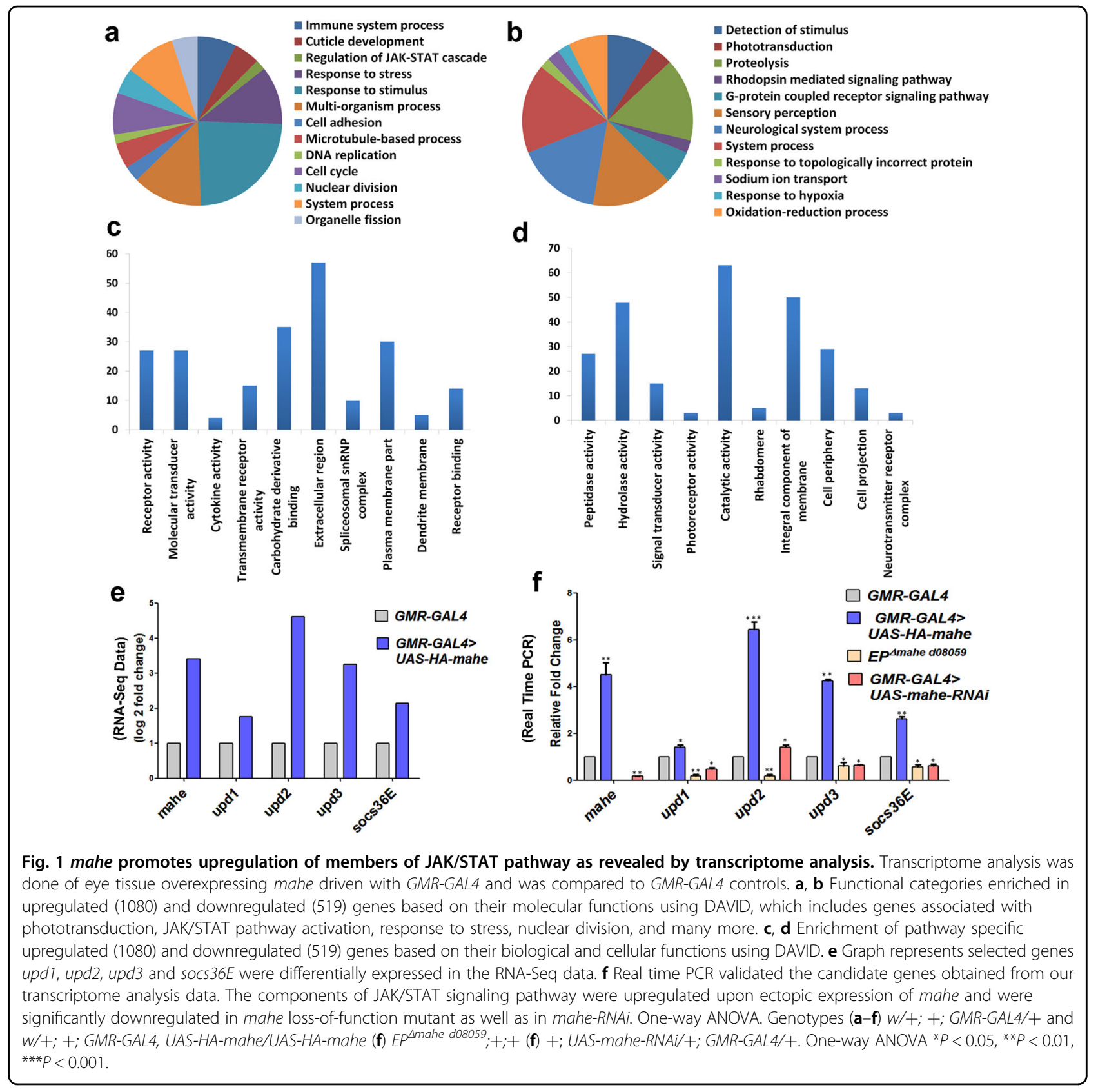

apoptotic phenotype. Coexpression of both mahe and upd2 exhibited severe disorganization of ommatidia with black necrotic patches that were observed in a few of the escapers, while most of the pupae failed to eclose and were dead. (Fig. $3 c-c^{\prime}$ ). This was in contrast to the rough and enlarged eye phenotype exhibited by overexpression of mahe and upd2 alone (Fig. 3a, $\mathrm{a}^{\prime}$ and $\mathrm{b}, \mathrm{b}^{\prime}$ ). Both, acridine orange and cleaved caspase immunostaining showed dramatic enhancement in apoptosis when upd 2 and mahe were coexpressed in the developing eyeantennal discs (Fig. 3h, $\mathrm{m}$ to $\mathrm{f}, \mathrm{k}$ and $\mathrm{g}, \mathrm{l}$ ). On similar lines, heterozygous upd 2 mutant (Fig. $3 \mathrm{~d}, \mathrm{~d}^{\prime}, \mathrm{i}, \mathrm{n}$ ) in combination with mahe overexpression completely rescued the apoptotic phenotype (Fig. 3e, $\mathrm{e}^{\prime}, \mathrm{j}$, o to a, $\mathrm{a}^{\prime}, \mathrm{f}, \mathrm{k}$ ). Quantitative analysis of cleaved caspase staining showed enhancement of apoptotic cells, when both upd2 and mahe were expressed together, whereas in contrary mahe induced phenotype was suppressed in heterozygous combination with upd2 loss of function allele (Fig. 3p).

We also observed that upd1 ligand that activates JAK/ STAT signaling ${ }^{17}$ also enhanced mahe induced apoptosis (Fig. S1). Our observation was supported by an independent transgenic RNA interference (RNAi) screen performed for mahe modifiers (unpublished Pandey and 


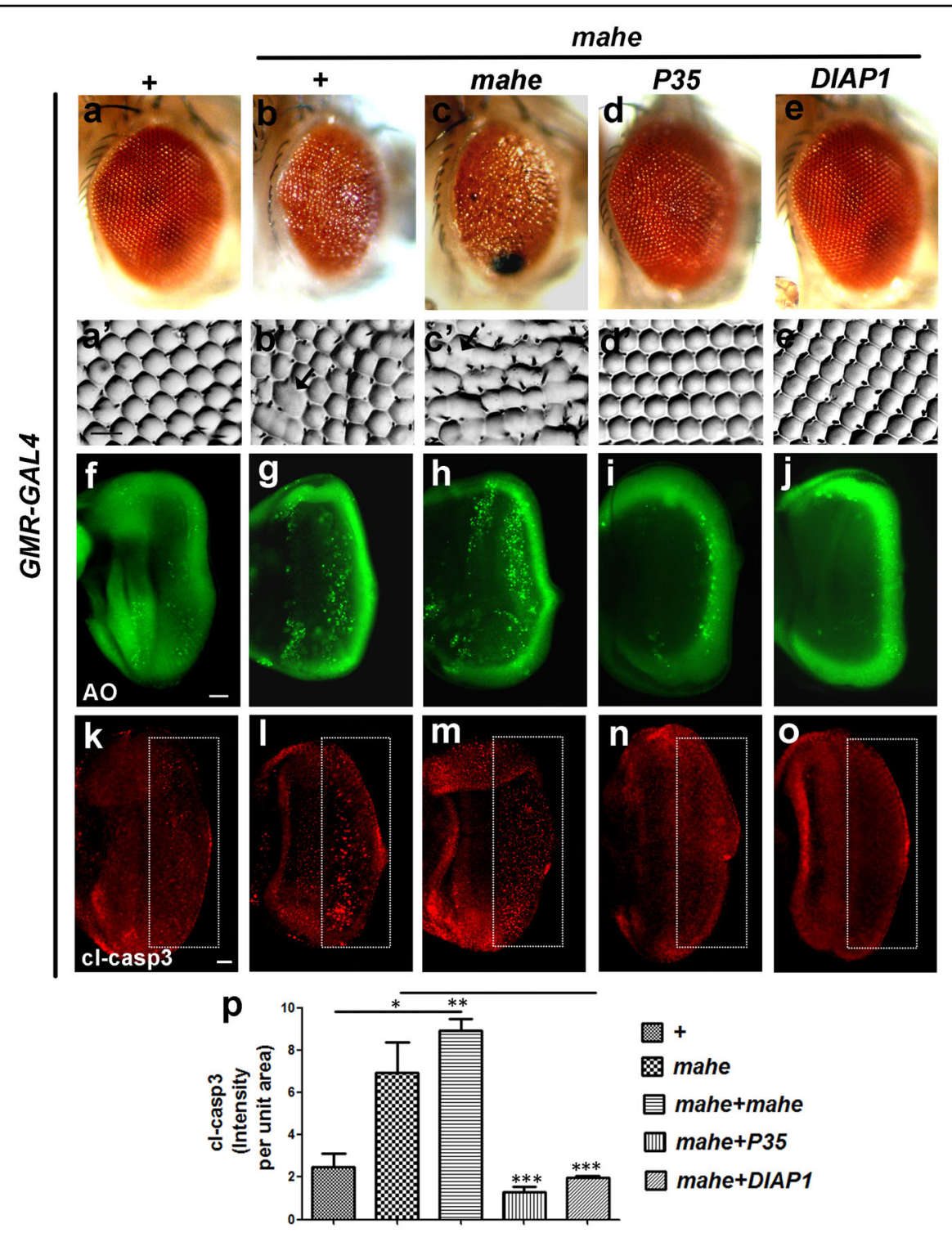

Fig. 2 Ectopic expression of mahe promotes caspase-dependent dosage sensitive cell death in eye tissue of Drosophila. a-e Images of Drosophila adult eyes. $\mathbf{a}^{\prime}-\mathbf{e}^{\prime}$ Eye imprints of adult eye. GMR-GAL4 was used as a control (a), or to drive expression of mahe (b-e), P35 (d), DIAP1 (e). a, a' Control adult eye showed normal ommatidium arrangement. b, b' GMR-GAL4 > mahe induced eye roughness with fused ommatidia (marked with arrow). c, $\mathbf{c}^{\prime}$ Ommatidial disarray and fusion were severely enhanced on increasing the dosage of mahe. $\mathbf{d}, \mathbf{d}^{\prime}$ mahe induced eye phenotype was suppressed by expression of P35, an inhibitor of effector caspase. e, $\mathbf{e}^{\prime}$ Similar to P35, inhibitor of initiator caspase DIAP1 suppressed the eye roughening and ommatidium disarrangement. $\mathbf{f}-\mathbf{j}$ Acridine orange staining of eye-antennal imaginal discs. $\mathbf{f}$ Acridine orange marks few dying cells in control eye-antennal disc. $\mathbf{g}, \mathbf{h}$ Number of acridine orange positive cells were enhanced upon mahe overexpression in dosage sensitive manner. $\mathbf{i}, \mathbf{j}$ P35 and DIAP1 suppressed cell death, shown by reduction in acridine positive nuclei. $\mathbf{k}-\mathbf{o}$ Caspase immunostaining of eye-antennal discs from third instar larvae (Compare area within rectangle). I, $\mathbf{m}$ Ectopic mahe leads to increase in number of caspase positive cells in comparison to wild type (k). $\mathbf{n}$, o Reduction in caspase positive cells were observed upon coexpression of P35 and DIAP1 along with mahe. (p) Graph represents intensity of clcasp3 per unit area showing mahe triggers caspase-dependent cell death, which was suppressed by P35 and DIAP1. One-way ANOVA. Scale bar in $50 \mu \mathrm{m}\left(\mathbf{a}^{\prime}-\mathbf{e}^{\prime}, \mathbf{f}-\mathbf{j}, \mathbf{k}-\mathbf{o}\right)$. Genotypes (a) w/t; +; GMR-GAL4/+ (b) w/t; +; GMR-GAL4, UAS-HA-mahe/+ (c) w/+; +; GMR-GAL4,UAS-HA-mahe/UAS-HAmahe (d) W/4; +; GMR-GAL4,UAS-HA-mahe/UAS-P35 (e) W/+; +; GMR-GAL4,UAS-HA-mahe/UAS-DIAP1. One-way ANOVA * $P<0.05,{ }^{* *} P<0.01,{ }^{* * *} P<$ 0.001 .

Mutsuddi). Downregulation of Chaperonin containing TCP1 subunit 7 (CCT7) gene, which is known to be a negative regulator of $u p d 1^{18}$ led to enhancement of apoptosis induced by mahe (Fig. S2) suggesting, a positive role of mahe induced JAK/STAT signaling in promoting cell death. Our genetic interaction studies are in agreement with our transcriptome data. Our results clearly display that ectopic expression of upd 2 dramatically 


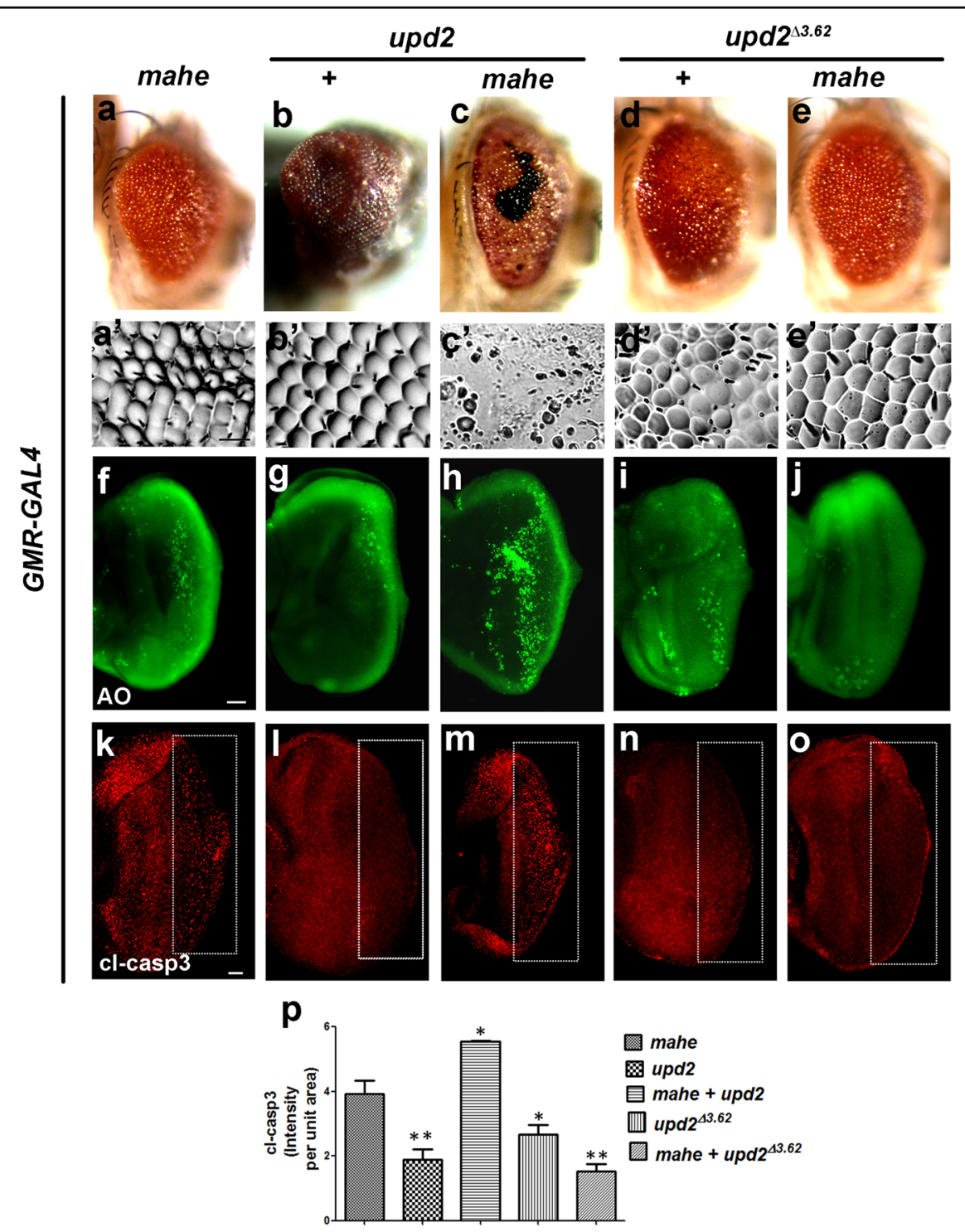

Fig. 3 Ectopic expression of upd2 enhances, while loss of upd2 suppresses mahe induced apoptosis in developing eye. a-e Images of Drosophila adult eye. $\mathbf{a}^{\prime}-\mathbf{e}^{\prime}$ Eye imprints of adult eye. GMR-GAL4 was used to drive the expression of UAS-mahe (a, $\left.\mathbf{c}, \mathbf{e}\right)$ or UAS-Upd2 (b, $\left.\mathbf{c}\right)$. $\mathbf{a}, \mathbf{a}^{\prime}$ GMRGAL4 > mahe promotes eye roughening. $\mathbf{b}, \mathbf{b}^{\prime}$ GMR-GAL4 driven upd2 resulted in severely overgrown eyes with regular patterned ommatidia. $\mathbf{c}, \mathbf{c}^{\prime}$ Coexpression of upd 2 and mahe leads to pupal lethality whereas few escapers showed drastic eye roughness, with loss of pigmentation, black necrotic patches and loss of ommatidia in comparison to ectopic expression of mahe alone. $\mathbf{d}$, $\mathbf{d}^{\prime}$ upd $2^{\Delta 3-62}$ a loss-of-function allele shows slightly disorganized and fused ommatidia along the dorsal side of adult eye. e, $\mathbf{e}^{\prime}$ upd2 $2^{43-62}$ in combination with GMR-mahe suppressed the disorganized adult eye phenotype. $\mathbf{f}-\mathbf{j}$ and $\mathbf{k}$-o Acridine orange and caspase staining (compare area marked within rectangle) in eye-antennal disc of third instar larvae. $\mathbf{f}, \mathbf{k}$ Acridine orange and caspase staining marked dying cells on ectopic expression of mahe. $\mathbf{g}$, I Fewer acridine orange and caspase positive cells were observed upon upd2 overexpression. $\mathbf{h}, \mathbf{m}$ Coexpression of upd2 and mahe enhanced cell death associated with mahe, which is reflected by increase in acridine orange and caspase positive cells when compared to mahe alone. $\mathbf{i}, \mathbf{n} u p d 2^{43-62}$ showed fewer acridine and caspase positive cells. $\mathbf{j}, \mathbf{o}$ upd $2^{13-62}$ rescued the mahe induced cell death, which is depicted by reduced number of acridine orange and caspase positive cells. $\mathbf{p}$ Graph represents $\mathrm{Cl}$-caspase intensity per unit area which shows upd2 overexpression promotes mahe induced cell death, and was significantly reduced in combination with upd2 mutant. One-way ANOVA. Scale bar in $50 \mu \mathrm{m}\left(\mathbf{a}^{\prime}-\mathbf{e}^{\prime}, \mathbf{f}-\mathbf{j}, \mathbf{k}-\mathbf{o}\right)$. Genotypes (a) $w /+;+;$ GMR-GAL4,UAS-HA-mahe/+ (b) W/t; UAS-upd2:GFP/+;GMR-GAL4/+ (c) w/+;UAS-upd2:GFP/+;GMR-GAL4,UAS-HA-mahe/+ (d) upd2 $13.62 /+;+;+$ (e) upd2 $13.62 /+;+; G M R-G A L 4$, UAS-HA-mahe. One-way ANOVA * $P<0.05,{ }^{* *} P<0.01,{ }^{* * *} P<0.001$.

enhances the apoptotic phenotype of mahe, while moderate enhancement of apoptosis was seen in combination of $u p d 1$ and mahe. Reduced levels of both upd1 and upd2 led to massive suppression of the mahe induced phenotype. This suggested a vital role of JAK/STAT signaling in mahe induced cell death. 
hopscotch transcripts coimmunoprecipitates with Mahe and this interaction leads to its stabilization

In Drosophila, upd acts through the Dome/Hop/ STAT92 signaling pathway for proper photoreceptor development ${ }^{14,19}$. We were further interested to check how mahe may be involved in altering JAK/STAT signaling. To determine the mechanism that connects mahe with JAK/STAT pathway-mediated apoptosis, we hypothesized that Mahe which encodes an RNA binding protein, might physically interact with the transcripts of any/ some of the major components of JAK/STAT pathway via its RNA binding domain thereby ectopically activating and enhancing JAK-STAT signaling output.

To test this hypothesis, immunoprecipitation of RNAprotein complex was carried out from fly head extracts using anti-HA beads to pull down HA-tagged Mahe, while RNA-protein extracted from GMR-GAL4 alone served as negative control. This was followed by RT-PCR using gene-specific primers for the members of JAK/STAT pathway, upd1, upd2, upd3, dome, hop, stat92E, and socs $36 E$. Interestingly, anti-HA pulled down HA-Mahe was positive only for the presence of hop transcripts that encode the tyrosine kinase involved in JAK/STAT signaling (Fig. $4 \mathrm{a}-\mathrm{c}$ ). Further, a complete absence of amplification with hop promoter-specific primers and positive amplification with hop exon-intron boundary-specific primers ensured the specificity of hop RNA and Mahe interaction. (Fig. 4d). This ruled out the interaction of Mahe RNA helicase with that of the promoter region or the DNA of hop.

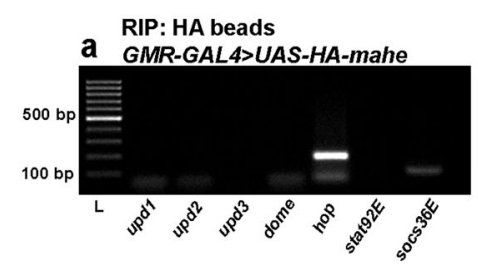

b RIP: HA beads

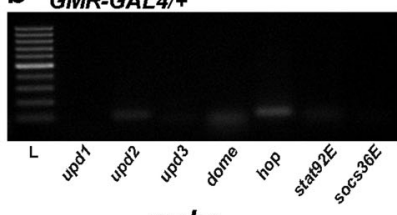

mahe
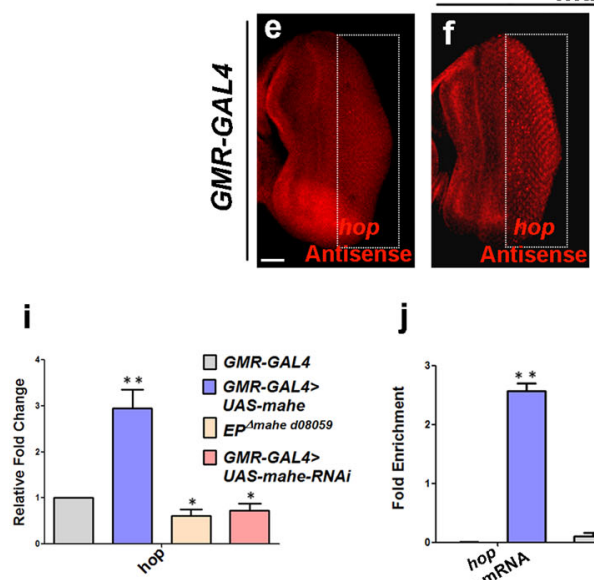

j

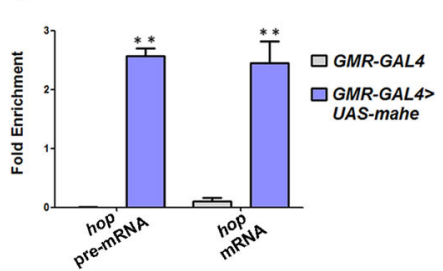

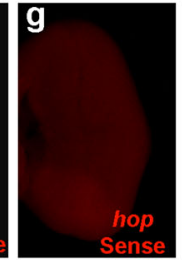

cDNA C $G M R-G A L 4 /+$

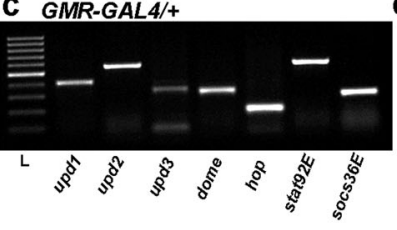

RIP: HA beads

d GMR-GAL4>

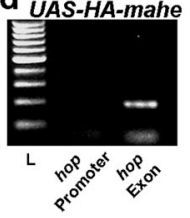

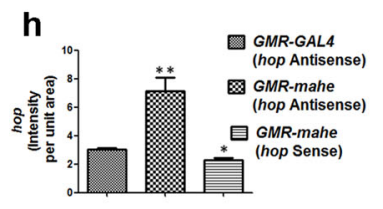

$\mathbf{k}$

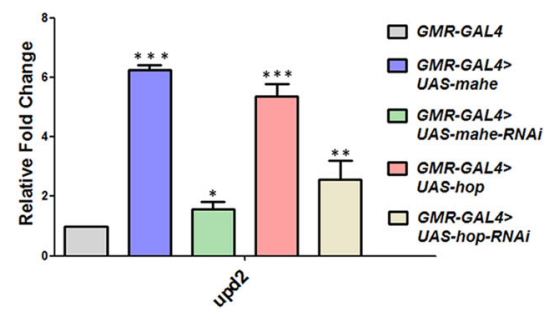

Fig. 4 Mahe directly binds to hop transcripts and increases/stabilizes its level leading to upregulation of upd2 ligand. a, b, d RNA immunoprecipitation was done using Anti-HA beads to immunoprecipitate HA-tagged-Mahe along with associated RNA-protein complex. a Protein lysates from GMR-GAL4 > UAS-HA-mahe was used for immunoprecipitation followed by RT-PCR with primers specific for components of JAK/STAT pathway. Out of the different components upd1, upd2, upd3, dome, hop,stat92E, and socs36E of JAK/STAT signaling, only hop was amplified indicating that Mahe binds with hop transcripts. b No amplification was observed in negative control in which GMR-GAL4/+ lysate was used for immunoprecipitation. c CDNA samples without RNA immunoprecipitation were used for positive control. $\mathbf{d}$ No amplification was observed with primer specific for hop promoter, while hop exon specific primers showed positive amplification. e-g RNA-FISH was done to check the level of hop transcripts in eye antennal discs. e FISH with hop-specific antisense riboprobe in wild-type eye antennal disc showed a very little signal. $\mathbf{f}$ Ectopic mahe expression revealed enhanced levels of hop transcript posterior to the morphogenetic furrow when compared to that of the control (area in rectangle). $\mathbf{g}$ hop sense riboprobe was used as negative control and no signal was seen in mahe overexpressed eye-antennal discs. $\mathbf{h}$ Graph represents hop intensity per unit area that clearly shows mahe overexpression leads to increase in hop transcript levels when compared to that of wild-type control. One-way ANOVA. i Quantitative real-time PCR shows increase in hop transcripts level upon mahe overexpression when compared to transcripts from GMR-GAL4 control tissue. One-way ANOVA. $\mathbf{j}$ Enrichment of both hop pre-mRNA and mRNA was observed by real-time PCR in RIP precipitate. One-way ANOVA. k Overexpression of hop alone leads to increase in upd2 transcript levels, similar to that of ectopic mahe. One-way ANOVA. Scale bar in $50 \mu \mathrm{m}(\mathbf{e}-\mathbf{g})$. Genotypes $(\mathbf{b}, \mathbf{c}, \mathbf{e}, \mathbf{h}, \mathbf{i}, \mathbf{j}, \mathbf{k}) W /+;+; G M R-G A L 4 /+(\mathbf{a}, \mathbf{d}, \mathbf{f}, \mathbf{g}, \mathbf{h}, \mathbf{i}, \mathbf{j}, \mathbf{k}) w /+;+; G M R-G A L 4$, UAS-HA-mahe/UAS-HA-mahe $(\mathbf{i}, \mathbf{k})$ W/4; UAS-mahe-RNAi/+; GMR-GAL4/+ (k) w/t; UAS-hop/+; GMR-GAL4/+ (k) w/+; UAS-hop-RNAi/+;GMR-GAL4/+. One-way ANOVA *P $<0.05$, ** $P<0.01,{ }^{* * *} P<0.001$. 
Additionally, fluorescent RNA in situ hybridization (FISH) with hop-specific antisense RNA probe was done in mahe overexpressed eye-antennal discs to visualize, whether the interaction of hop transcripts with Mahe led to any change in the turnover rate of hop transcripts. Increase in hop transcript levels was seen in mahe overexpressed discs in comparison to the wild-type control discs, whereas no signal was observed with the hop sense probe (Fig. 4e-h). For unchanged control $h s r \omega$ probe was used, and no change in transcript levels was observed in eye antennal discs overexpressing mahe when compared to GMR-GAL4 control (Fig. S3). This observation clearly signifies that recruitment of hop transcripts in the Mahe protein complex leads to stabilization and increase in hop RNA levels. RT-PCR analysis was also in agreement with our FISH data (Fig. 4i). The next question was whether mahe enhanced the levels of processed hop transcripts or pre-mRNA. RT-PCR using hop premRNA and mature RNA-specific primers identified the presence of both types of RNAs in the ribonucleoprotein complex (Fig. 4j). It is likely that the interaction of hop transcripts with Mahe RNA helicase reduces the turnover rate of transcripts and stabilizes it, thus resulting in elevated levels of hop RNA. We further hypothesized that ectopically elevated hop transcripts might stimulate upregulation of upd2 ligand which in turn promotes a positive feedback signal for ensuring prolonged JAK/STAT activation. Our proof of principle was strengthened by the elevated levels of upd2 RNA in tissues where hop was overexpressed (Fig. 4k).

To further establish the link between active hop and mahe, we carried out genetic interaction with hyperactive JAK Kinase UAS-hop ${ }^{(\text {Tum-l) }}$ which carries a dominant mutation in hop that activates Stat92E and with UAS-hop-RNAi. Coexpression of GMR-GAL4 driven mahe and UAS-hop (Tum-l) resulted in an enhanced rough eye phenotype as compared to that of expressing both the components by themselves (Fig. 5c, $c^{\prime}$ to a, $a^{\prime}$ and b, b'). Further, extensive cell death was detected posterior to the morphogenetic furrow that was depicted by acridine orange and anti-caspase antibody immunostaining in eye-antennal discs with overexpressed hop and mahe (Fig. 5h, $\mathrm{m}$ to $\mathrm{f}, \mathrm{k}$ and $\mathrm{g}, \mathrm{l}, \mathrm{p}$ ). In line with above genetic interaction, conversely downregulation of hop led to suppression of mahe induced apoptotic phenotype (Fig. 5e, e $e^{\prime}, j$, o to a, a', f, k and d, d', i, n). Thus, our molecular and genetic interaction studies suggest that interaction of Mahe with hop RNA stabilizes the hop transcripts, leading to the activation of JAK/STAT pathway, which in turn induces apoptosis.

\section{Increase in both cytoplasmic and nuclear Stat92E levels indicates activation of JAK/STAT pathway that ultimately leads to hid mediated apoptosis}

To investigate the role of mahe in activating JAK/STAT signaling we checked the status of the most downstream component Stat92E, a transcription factor required for
JAK/STAT signaling. To test this, we utilized $10 X$ Stat92E-GFP line containing five tandem repeats of socs $36 E$ binding sites, a transcriptional reporter for JAK/ STAT signaling ${ }^{20}$. As predicted, we observed a surge in Stat92E-GFP reporter activity upon ectopic expression of mahe, when compared to that of the basal Stat92E reporter gene expression (Fig. 6a-d). This supported our transcriptome data where we saw elevated socs36E transcripts, which is a downstream target of Stat92E (Fig. 1e, f). The above results further strengthened our findings that JAK/STAT pathway is activated and upregulated by mahe.

We argued that an elevated expression of Stat92E protein can be correlated with increase in activation of JAK/ STAT pathway. We assessed the expression of Stat92E protein in gain-of-function mahe clones to check whether the levels of STAT92E protein were altered in the somatic clones. The stock $y w$ hsp70-flp; Act FRT $y^{+}$FRT-GAL4 $U A S-G F P /+$; +, was used to generate clonal cells in the salivary gland, in which mahe levels were enhanced with UAS-HA-mahe (Fig. 6e-l). The somatic clones with enhanced Mahe expression were marked with GFP (Fig. 6i-l). A substantial increase in Stat92E expression in the nucleus and cytoplasm was observed in UAS-HA-mahe clones, whereas in the non-clonal cells, the fluorescent signal was comparatively lower (Fig. $6 \mathrm{e}-\mathrm{h}, \mathrm{m}$ ). We also made an effort to generate mahe loss-of-function mutant clones using FLP-FRT system; however, mutant clones of mahe did not survive and hence we were unable to carry out mahe loss of function analysis. However, the accumulation of Stat92E protein in mahe overexpression clones is a strong proof that mahe leads to activation of JAK/STAT pathway.

It has been previously reported that upd via JAK/STAT signaling promotes apoptosis through hid in supernumerary polar cells during Drosophila oogenesis ${ }^{21}$. Suppression of mahe rough eye phenotype by DIAP1 (Fig. 2), led us to examine the effect of proapoptotic genes in mahe mediated apoptosis. H99 mutant in which all the three genes hid, rpr and grim are deleted, in a heterozygous combination led to suppression of ectopic mahe induced phenotype. We then carried out genetic interaction analysis with mutants of proapoptotic genes. It was observed that mutations in hid, but not in rpr and grim suppressed mahe induced rough eye phenotype (Fig. $6 \mathrm{n}-\mathrm{r}$ and n'- r'). In addition, a 3 fold increase in hid transcript levels were observed with mahe overexpression, supporting the involvement of hid in mahe induced cell death (Fig. 6s).

Next, we quantified hid transcript levels after blocking JAK/STAT activity. Lowering levels of Stat $92 E$ via RNAi in mahe overexpression background, led to a 2.5 fold decrease in hid transcripts in comparison to transcripts from mahe overexpressed tissue (Fig. 6s). This clearly 


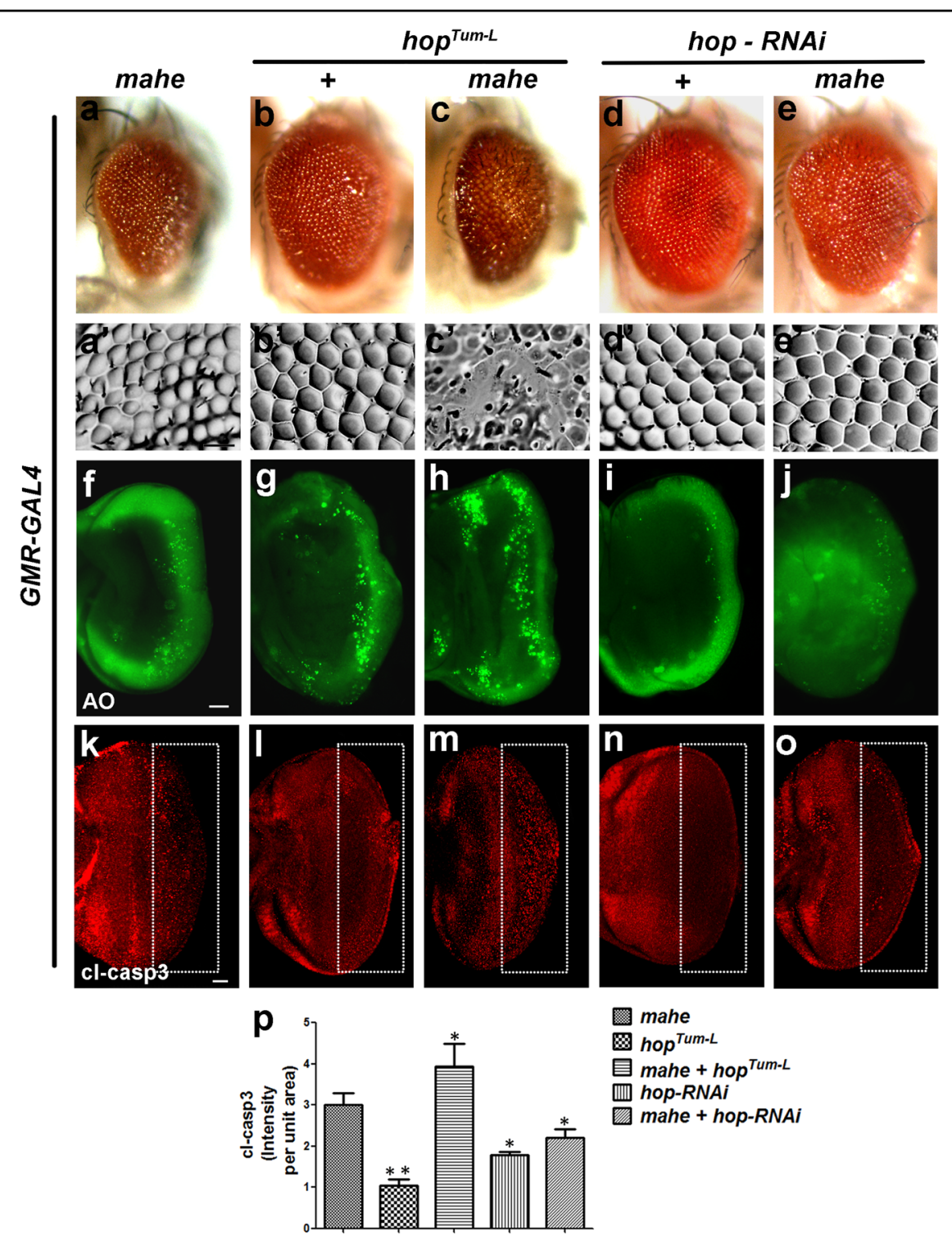

Fig. 5 hopscotch enhances mahe induced cell death. (a-e) Images of Drosophila adult eye. $\mathbf{a}^{\prime}-\mathbf{e}^{\prime}$ Adult eye imprints. a, c, e GMR-GAL4 was used to drive UAS-mahe or (b, c) UAS-hop ${ }^{(T u m-1)}$ (d, e) UAS-hop-RNAi. a, a' Ectopic mahe expression promotes eye roughening. $\mathbf{b}$, $\mathbf{b}^{\prime}$ Overexpression of constitutive active form of hop results in slight ommatidial disorganization. $\mathbf{c}, \mathbf{c}^{\prime}$ Coexpression of hop and mahe enhanced the rough eye phenotype compared to mahe alone. $\mathbf{d}$, $\mathbf{d}^{\prime}$ Reduction of hop levels by RNAi mediated knockdown leads to no observable change in eye roughening phenotype. $\mathbf{e}, \mathbf{e}^{\prime}$ RNAi mediated depletion of hop rescued the mahe induced eye roughness. $\mathbf{f}-\mathbf{j}, \mathbf{k}-\mathbf{o}$ Acridine orange and caspase staining in eye antennal disc. $\mathbf{f}, \mathbf{k}$ Acridine and caspase positive cells show cell death induced by mahe. $\mathbf{g}$, I hop ${ }^{\text {Tum-l }}$ overexpression showed few dying cells. $\mathbf{h}$, $\mathbf{m}$ Coexpession of hop ${ }^{\text {Tum-l }}$ and mahe enhanced cell death in comparison to mahe alone. $\mathbf{i}, \mathbf{n}$ No dying cells were observed on downregulating the level of hop. $\mathbf{j}$, o Downregulation of hop rescued the mahe induced cell death as depicted by acridine orange and cl-caspase staining. $\mathbf{p}$ Graph represents $\mathrm{Cl}$ caspase intensity per unit area which shows activated hop ${ }^{\text {Tum-l }}$ promotes mahe induced cell death, which was significantly reduced in combination with hop-RNAi. One-way ANOVA. Scale bar in $50 \mu \mathrm{m}(\mathbf{f}-\mathbf{j}, \mathbf{k}-\mathbf{o})$. Genotypes (a) $w_{;}+;$GMR-GAL4,UAS-HA-mahe/+ (b) w; UAS-hop ${ }^{\text {Tum-l } /} /+;$;GMR-GAL4/+ (c)

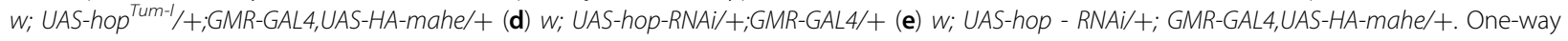
ANOVA ${ }^{*} P<0.05,{ }^{*} P<0.01,{ }^{* *} P<0.001$.

indicated that mahe positively regulates JAK/STAT pathway, which ultimately modulates the levels of hid transcripts and lowering of the downstream effector Stat $92 E$ alone was sufficient to rescue the hid mediated apoptosis.
Based on our findings we put forward a hypothetical model (Fig. 7), where we propose that Mahe regulates JAK/STAT signaling by directly interacting with hop tyrosine kinase transcripts leading to its stabilization and this cascade the activation of downstream effector 


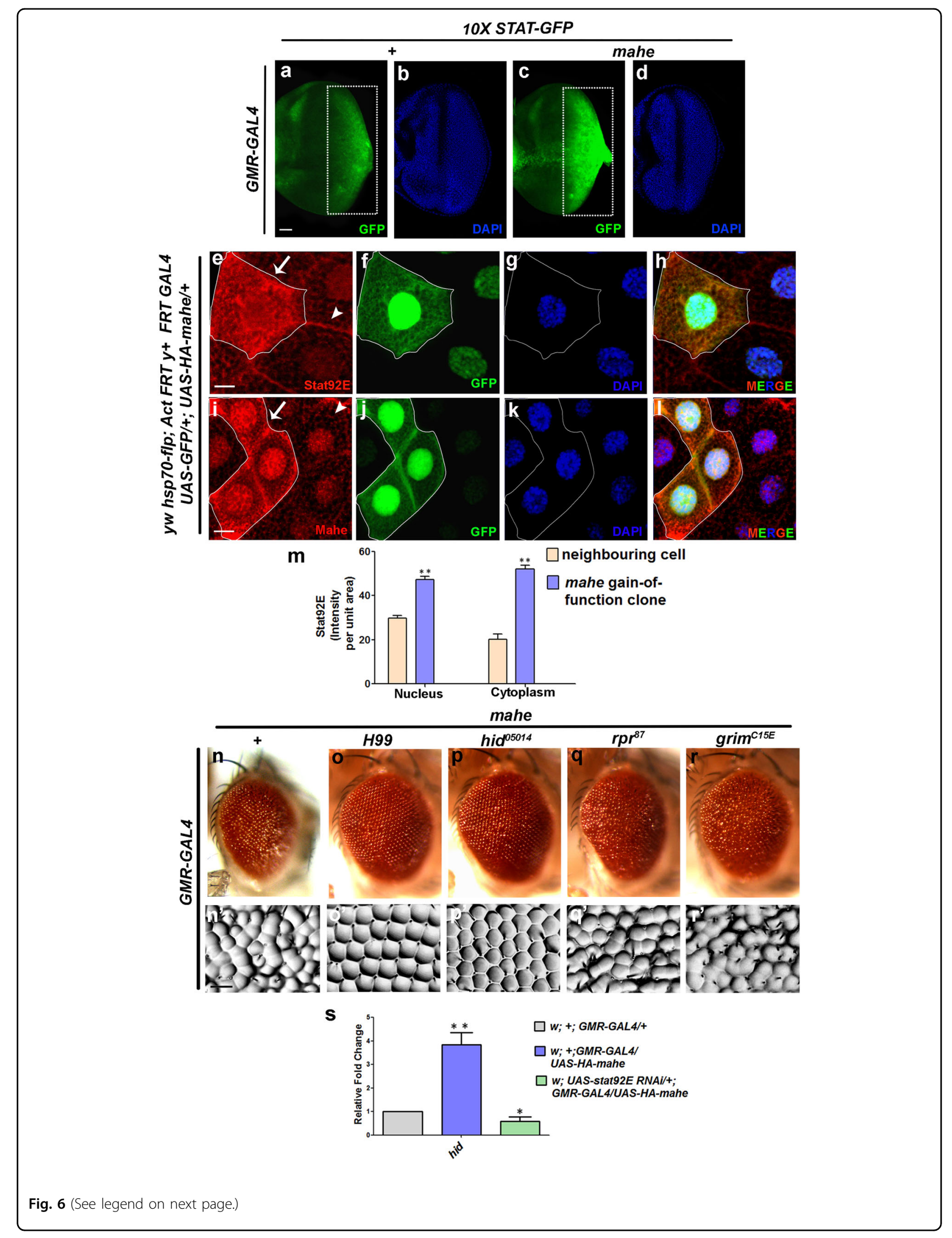


(see figure on previous page)

Fig. 6 JAK/STAT pathway activation by ectopic mahe induces hid dependent apoptosis. a-d Activation of JAK/STAT pathway was detected by 10X STAT92E-GFP reporter in eye-antennal discs. a, c Significant increase in GFP level was observed in GMR-GAL4 driven mahe eye antennal disc when compared to the reporter line alone, indicating activation of JAK/STAT signaling (compare area in rectangle). $\mathbf{b}$, $\mathbf{d}$ DAPI was used to mark the nucleus. e-I Gain of function clone of mahe displays enhanced Stat92E activity. Gain of function clones of mahe using UAS-HA-mahe flies were generated with FLP/FRT system in salivary gland. $\mathbf{f}$, j GFP positive and non-GFP cells mark mahe gain-of-function clones (marked with arrow) and wild-type cells (marked with arrowhead), respectively. e Level of Stat92E the transcription factor for JAK/STAT signaling was enhanced in mahe gain-of function somatic clones when compared to wild-type neighboring cells. i Clonal area marked with GFP positive cells, shows elevated level of Mahe in the gain-of-function clones. $\mathbf{g}, \mathbf{k}$ DAPI was used to mark the nucleus. $\mathbf{h}$, I Fourth column represents merged image with DAPI staining. $\mathbf{m}$ Graph represents Stat92E intensity per unit area which shows marked enhancement in the level of Stat92E in nucleus and cytoplasm, in mahe overexpressing clones when compared to neighboring cells. One-way ANOVA. $\mathbf{n}, \mathbf{n}^{\prime}$ Ectopic expression of mahe results in rough eye phenotype. $\mathbf{0}, \mathbf{o}^{\prime}$ and $\mathbf{p}, \mathbf{p}^{\prime}$ mahe induced rough eye phenotype was rescued by $\mathrm{H} 99$ and hid mutant, but not with $r p r$ and grim mutants $\left(\mathbf{q}, \mathbf{q}^{\prime}\right.$ and $\left.\mathbf{r}, \mathbf{r}^{\prime}\right)$. $\mathbf{s}$ Real time PCR showed a threefold increase in hid transcript levels upon ectopic expression of mahe, while downregulation of JAK/STAT pathway by stat92E-RNAi rescued the hid levels. One-way ANOVA. Scale bar in $50 \mu \mathrm{m}(\mathbf{a}-\mathbf{d}, \mathbf{e}-\mathbf{l})$. Genotypes (a, b) w/t; 10X STAT-GFP/+, GMR-GAL4/+ (c, d) W/+; IOX STAT-GFP/+; GMR-GAL4, UAS-HA-mahe/+ (n) W/+; +; GMR-GAL4,UAS-HA-mahe/+ (o) W/+; +; GMR-GAL4,UAS-HA-mahe/H99 (p) W/+; +; GMRGAL4,UAS-HA-mahe/hid ${ }^{05014}$ (q) W/+; +; GMR-GAL4,UAS-HA-mahe/rpr ${ }^{87}$ (r) w/t; +; GMR-GAL4,UAS-HA-mahe/grim ${ }^{\text {C15E }}$. One-way ANOVA * $P<0.05$, ** $P<0.01,{ }^{* * *} P<0.001$.

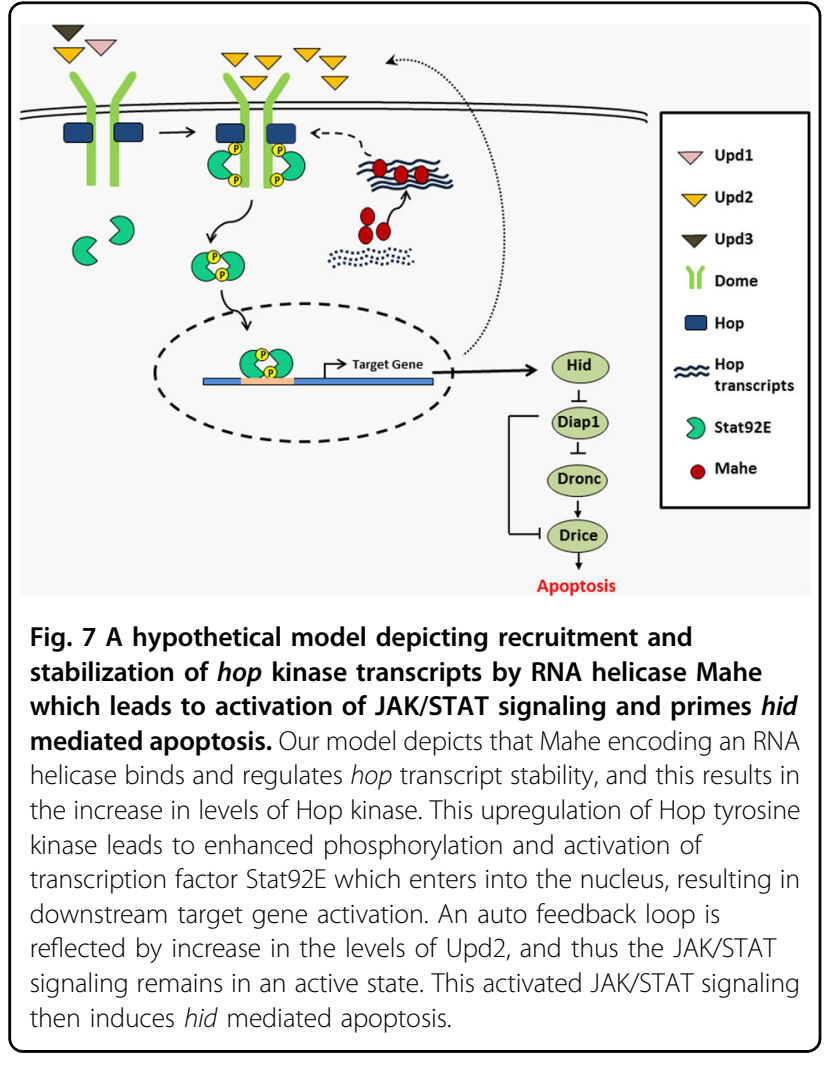

molecules in this pathway. This in turn leads to activation of proapoptotic gene hid, along with a surge in apoptosis.

\section{Discussion}

A number of reports have shown that Decapentaplegic (Dpp), Hedgehog (Hh), Notch, JAK/STAT and Wingless (Wg) signaling pathways are major regulators of development and differentiation of photoreceptor neurons in Drosophila $^{22-28}$. Dysregulation of JAK/STAT pathway has been implicated in a wide variety of neurological disorders like the glial tauopathy and Parkinson's Disease ${ }^{29-31}$. We have recently reported that mutation in $D D X 59$, the human homolog of maheshvara (mahe), is associated with neurological abnormalities and is crucial for development of the nervous system ${ }^{11}$.

In this report we have elucidated a novel link between mahe and JAK/STAT signaling, suggesting the significance of mahe in regulation of JAK/STAT signaling in the photoreceptor neurons of Drosophila. Our transcriptome analysis has identified differentially regulated genes that are predominantly related to neuronal function, phototransduction, stress response and regulation of cell signaling. Interestingly, by quantitative-PCR analysis, we found that all the ligands and downstream effector of JAK/STAT pathway were significantly lowered in mahe loss-of-function mutant and in mahe-RNAi. Similarly, upregulation of the pathway components suggested ectopic activation of the JAK/STAT signaling by overexpressed mahe, which was in agreement with the gene ontology analysis of transcriptome data.

JAK/STAT signaling is an important pathway required for Drosophila eye development and for proper axon targeting in a cell autonomous manner during photoreceptor formation ${ }^{32}$. Further, genetic studies have previously shown that overexpression of upd and hop ${ }^{\text {Tumorous-lethal }}$ which encodes constitutively active form of hop results in dramatically overgrown adult eyes. In addition, downregulation of JAK/STAT pathway by hypomorphic upd mutant and dominant negative form of dome $e^{\Delta c y t}$ leads to small or ablated eyes ${ }^{19,22,33-35}$. However, in contrast in lobe mutants, ectopic upd expression is known to activate JAK/STAT pathway and induce apoptosis, which leads to small eye phenotype ${ }^{36}$. Taken together these findings suggest that JAK/STAT pathway can induce both context-dependent proliferation as well as apoptosis and is needed for proper eye development in Drosophila. Although both upd2 and upd 3 are ligands for JAK/STAT pathway but their role in Drosophila 
photoreceptor development, specifically cell death has not been investigated. Here, we observed that coexpression of both mahe and upd2 along with other components of JAK/STAT pathway results in massive apoptosis. Our findings reveal that mahe activated JAK/STAT signaling can induce apoptosis in Drosophila photoreceptors thus showing context-dependent role of JAK/STAT pathway during development. Conversely, loss of JAK/STAT signaling components leads to rescue in the mahe-induced apoptosis.

RBPs are known to modulate post-transcriptional regulation, turnover, localization, and translational control of mRNA. Reports have shown that RBPs can act in both positive or negative manner to stabilize $\mathrm{mRNA}^{37,38}$. TTP and KSRP destabilizes several mRNA like $c$-fos, TNF $\alpha$ and $C O X-2$, however $H u R$ acts positively to maintain their stability $^{39-42}$. A recent report revealed that RNA binding protein Musashi 2 (MSI2) regulates IL-6 signal transducer (IL6ST) and promotes its degradation by binding to $3^{\prime}$ UTR of mRNA. IL6ST cytokine receptor in turn after forming complex with kinases affects the phosphorylation of STAT3 and other mitogen-activated protein kinase $\mathrm{ERK}^{43}$. Similarly, in order to understand the mechanism of ectopic JAK/STAT activation, we argued whether Mahe a DEAD box RNA helicase might interact directly with the transcripts of the components of JAK/STAT transduction cascade to regulate the pathway. Interestingly, hop transcripts were found to be associated with Mahe-containing RNA-protein complex. We propose a model in which this interaction may lead to decrease in the turnover rate of transcripts, and thus leads to stabilization and elevation in hop transcripts. The elevated JAK is activated possibly via interaction with the receptor and ligand Upd2 in response to ectopic mahe. Mechanism that could account for activation of the pathway is probably via augmented phosphorylation and activation/ stabilization of downstream transcription factor Stat92E, as seen by its enhanced cytoplasmic to nuclear localization. Next, we hypothesized that this interaction can result in enhanced upd2 RNA levels, which is further supported by a significant increase in upd 2 transcript levels upon expression of hop alone. Our prediction that mahe activates downstream targets of JAK/STAT signaling was supported by our reporter gene analysis using $10 \mathrm{X}$ Stat92E GFP line. Further, a massive increase in both cytoplasmic and nuclear Stat92E transcription factor was seen in mahe gain-of-function somatic clone. Activation of the JAK/STAT pathway was further reflected by the increase in transcription of downstream target of socs $36 E$. The above finding was supported by a report where oral infection initiates immune response in the gut of Drosophila followed by upregulation of JAK/STAT signaling, and the pathway activity was assessed by transcript levels of $\operatorname{socs} 36 E^{44}$. Based on these findings we propose that
Mahe and hop interaction upregulates the JAK/STAT pathway via an auto feedback loop.

In summary, we report that mahe induces JAK/STAT signaling mediated apoptosis during Drosophila photoreceptor development. We put forth a novel mechanism in which hop is post-transcriptionally regulated by a DEAD box RNA helicase Maheshvara, which ultimately results in activation of JAK/STAT signaling mediated apoptosis. As deregulated JAK/STAT signaling and apoptosis have been linked to many human diseases, the identification of a novel regulator of this pathway will lead to a better understanding of development and disease. Identification of Maheshvara as a novel modulator of JAK/STAT pathway has opened up a novel platform for understanding the role of RNA helicase associated cell death which may also hold good for human disorders as well.

\section{Material and methods Drosophila genetics}

All the fly stocks were maintained on standard cornmeal/yeast/molasses/agar medium at $25^{\circ} \mathrm{C} . w^{1118}$ was used as the wild-type control. UAS-HA-mahe ${ }^{10}$ and $E P^{\Delta m a h e}$ d0805911 were generated in our lab. UAS-upd2GFP and $u p d 2^{\triangle 3-62}$ were kind gift from Prof. James Castelli-Gair Hombría. UAS-mahe-RNAi (v109465), UAShop-RNAi, UAS-stat92E-RNAi, UAS-upd1-RNAi, UASCCT7-RNAi were obtained from VDRC.GMR-GAL4, UAS-DIAP, UAS-P35, UAS-upd1, UAS-hop, 10X STATGFP, H99, hid $^{05014}, \mathrm{rpr}^{87}$, grim $^{\text {C15E }}$, stocks were obtained from Bloomington Drosophila Stock Center. All the crosses were performed at $25^{\circ} \mathrm{C}$, unless mentioned otherwise. To generate mahe gain-of-function clones females of hsp70-flp; Act FRT $y^{+}$FRT-GAL4 UAS-GFP/+; $+/+$ were crossed to UAS-HA-mahe males. Heat shock was given at $37^{\circ} \mathrm{C}$ for $10 \mathrm{~min}$ at $24 \mathrm{~h} \mathrm{AEL}$ and the third instar larvae were analyzed for GFP marked clones.

\section{Eye imprints}

Eye imprints using nail polish were prepared for analysis of ommatidial defects and were examined under differential interference contrast (DIC) optics in a Nikon Eclipse 80i microscope.

\section{Acridine orange staining}

To observe the extent of apoptosis we used the vital dye acridine orange (AO). Eye-antennal discs from larvae of desired genotypes were dissected in phosphate buffer saline (PBS) $\left(130 \mathrm{mM} \mathrm{NaCl}, 7 \mathrm{mM} \mathrm{Na} \mathrm{HPO}_{4}, 3 \mathrm{mM}\right.$ $\mathrm{KH}_{2} \mathrm{PO}_{4}, \mathrm{pH}$ 7.4) and stained with $1 \mu \mathrm{g} / \mu \mathrm{l}$ of Acridine orange (AO) in PBS for $3 \mathrm{~min}$. Followed by two washes with PBS and were finally mounted in PBS. These were than immediately viewed under Nikon Eclipse 80i microscope. 


\section{Immunostaining}

Immunostaining was performed in various tissues dissected from third instar larvae. Larvae were dissected in PBS ( $\mathrm{pH}$ 7.4) and immunostaining was done as described previously ${ }^{10}$. To mark the nuclei, staining with 4', 6- diamidino-2-phenylindole dihydrochloride (DAPI) $(1 \mu \mathrm{g} / \mathrm{ml})$ was done. Tissues were mounted in DABCO. All slides were observed under LSM 780 laser scanning confocal microscope Zeiss (Carl Zeiss), Thornwood, NY. The images were further processed with Adobe Photoshop 7. The following primary antibodies were used in this study: rabbit anti-Mahe (1:300) generated in our laboratory, rabbit anti-cleaved Caspase 3 (1:100) (Cell Signalling Technology), rabbit antiStat92E (1:200) a kind gift from Erika Bach. Secondary antibody used was Alexa Fluor 555 conjugated goat antirabbit IgG (1:200). Intensity profile graphs were made by using Image J and Graph Pad Prism 5 software.

\section{RNA extraction and Real-time PCR}

Total RNA was extracted using Trizol reagent (Invitrogen), from adult fly heads of desired genotypes. To remove genomic DNA contamination the extracted RNA was treated with RNase free DNaseI for $30 \mathrm{~min}$ at $37^{\circ} \mathrm{C}$. Reverse transcription was performed with a cDNA synthesis kit (Applied Biosystems, Foster City, CA), using aliquots of total RNA extracted. Real-time quantitative PCR was performed to check the expression of desired genes of interest. Real-time PCR reactions were performed using the ABI 7500 sequence detection system (Applied Biosystems) with SYBR Green PCR Master Mix (Thermo Scientific). The relative quantity of amplified cDNA/DNA corresponding to the gene was determined using the $\Delta \Delta C$ t method and normalized for expression of rps17 in each sample ${ }^{45}$. The graph was prepared using Graph Pad Prism 5 software.

Primers used for the study are as follows:

mahe forward primer: 5'-TTCGTGCGTTGGCCCTTG TTATTG-3'

mahe reverse primer: 5'-GCTGGGCATCGAACGAGC AAG-3'

upd 1 forward primer: $5^{\prime}$ - ATTGCCCTAAAGCGCTGG TACCG-3'

upd1 reverse primer: $5^{\prime}$ - GTAGTAGTGGTGCTTCAC AAAGC-3'

upd2 forward primer: 5' - GTGAAGCTAAAGACTTG-3' upd2 reverse primer: 5'- TCAAGACTCATTGGATC CGCCAT-3'

upd3 forward primer: 5'-TGCCCCGTCTGAATCTCA CT- $3^{\prime}$

upd3 reverse primer: 5'-GTGAAGGCGCCCACGTAA-3'

hop forward primer: 5'- GGGTATCTACATCAGATTG $\mathrm{TC}-3^{\prime}$ hop reverse primer: $5^{\prime}$-GCATTCACGCACAATATAG C- $3^{\prime}$

hop promoter forward primer: 5'-CAAGAATATAGAC GCCATAGAGC-3'

hop promoter reverse primer: $5^{\prime}$-GTCATCGATTGTCC AATA ACCTG-3'

socs36E forward primer: 5' - GCTGCCAGTCAGCAAT ATGT-3'

socs $36 E$ reverse primer: $5^{\prime}-$ GACTGCGGCAGCAACTG $\mathrm{T}-3^{\prime}$

hid forward primer: 5'-AGC GTCTGCAGGAGTTCA AT-3'

hid reverse primer: $5^{\prime}$-CTTCGCCTTTTGTCGTTCT C-3'

rps17 forward primer: 5'-AAGCGCATCTGCGAGGA G-3'

rps17 reverse primer: 5'-CCTCCTCCTGCAACTTGA TG-3'.

\section{Transcriptome analysis}

Total RNA was isolated from 200 adult fly heads using standard Trizol method (Sigma) from adult fly heads of GMR-GAL4 driven UAS-mahe and GMR-GAL4 alone which served as control for comparison. Libraries were made using standard protocol of TrueSeq RNA sample Prep kit v2. Libraries were then sequenced by the pairedend reads using Illumina HiSeq2500 platform and the resulting sequencing reads were aligned to the reference Drosophila melanogaster genome downloaded from Ensemble database (ftp://ftp.ensembl.org/pub/release81/fasta/drosophila_melanogaster/dna/Drosophila_melanogaster.BDGP6.dna.toplevel.fa.gz). The alignment was performed by STAR program (version=STAR_2.4.1d). Further, the aligned reads were used for estimating expression of transcripts using cufflinks program (version: cufflinks-2.2.1). The expression values are reported in FPKM (Fragment per kilo per million) units for each of the genes and transcripts. Differential expression analysis was performed using cuffdiffv2.2.1. Gene ontology analysis was done using DAVID 6.8 (The Database for Annotation, Visualization and Integrated Discovery).

\section{Immunoprecipitation of RNA-protein complex}

Protein lysates were prepared by homogenizing 200 adult heads from 1 day old flies of GMR-GAL4 driven UAS-mahe and GMR-GAL4 alone which served as control for comparison in lysis Buffer $(100 \mathrm{mM} \mathrm{KCl}, 5 \mathrm{mM} \mathrm{MgCl} 2,10 \mathrm{mM}$ HEPES, pH 7.0, 0.5\% Nonidet P-40, 1 mM DTT, $100 \mathrm{U}$ $\mathrm{ml}^{-1}$ RNase inhibitor (NEB), $2 \mathrm{mM}$ vanadyl ribonucleoside complexes solution (Sigma- Aldrich, $25 \mu \mathrm{ml}^{-1}$ protease inhibitor cocktail (Roche). Supernatant was collected and centrifuged at $12,000 \mathrm{~g}$ for $20 \mathrm{~min}$ at $4{ }^{\circ} \mathrm{C}$. Equilibration of anti-HA agarose beads was done in lysis buffer. Protein 
lysates were precleared by incubating with $20 \mu \mathrm{l}$ of anti-HA agarose beads for $2 \mathrm{~h}$ at $4{ }^{\circ} \mathrm{C}$. For immunoprecipitation, $50 \mu \mathrm{l}$ of anti-HA beads were used for every $250 \mu \mathrm{l}$ of protein lysate and were incubated for $5 \mathrm{~h}$ at $4{ }^{\circ} \mathrm{C}$ to pull down the desired protein of interest. This was followed by brief centrifugation to collect the beads. These beads were washed with $0.5 \mathrm{ml}$ of polysome lysis buffer thrice by centrifugation at $2000 \mathrm{~g}$ for $5 \mathrm{~min}$ at $4{ }^{\circ} \mathrm{C}$. Washed beads were resuspended in $100 \mu \mathrm{l}$ of lysis buffer with $30 \mu \mathrm{g}$ of proteinase $\mathrm{k}$ and $0.1 \% \mathrm{SDS}$ followed by heating at $50^{\circ} \mathrm{C}$ for $30 \mathrm{~min}$ in order to degrade the protein. Total RNA was extracted using Trizol reagent. Upper aqueous phase having RNA was recovered by centrifugation and to it $10 \mu$ of yeast tRNA $\left(1 \mathrm{mg} \mathrm{ml}^{-1}\right), 12 \mu \mathrm{l}$ of $3 \mathrm{M}$ sodium acetate and $250 \mu \mathrm{l}$ of ethanol were added per $100 \mu \mathrm{l}$ of aqueous phase and kept at $-20^{\circ} \mathrm{C}$ overnight for precipitation. This was followed by centrifugation at $12,000 \mathrm{~g}$ for $20 \mathrm{~min}$ at $4{ }^{\circ} \mathrm{C}$, ethanol was removed and the pellet rinsed with $70 \%$ ethanol followed by air drying until all the liquid evaporated. The pellet was resuspended in nuclease free MQ water. cDNA was synthesized from total RNA using M-MuLV Reverse Transcriptase (New England Biolabs) as per the manufacturer's instructions. RT-PCR was performed using primers for upd1, upd2, upd3, dome, hop, stat92E, and socs $36 E$ listed above.

\section{RNA: RNA in situ hybridization}

RNA-RNA in situ hybridization was carried out in tissue from third instar larvae. Larvae were dissected and RNA:RNA in situ hybridization was carried out as described earlier ${ }^{37}$. Hybridization was done with $100 \mathrm{ng}$ hop antisense riboprobe in hybridization buffer at $50^{\circ} \mathrm{C}$ for $12-16 \mathrm{~h}$. hop sense riboprobe was used as negative control and was hybridized at $50^{\circ} \mathrm{C}$ for $12-16 \mathrm{~h}$. hsrw antisense riboprobe served as positive control and was hybridized for $12-16 \mathrm{~h}$ at $50{ }^{\circ} \mathrm{C}$. For fluorescent detection of the riboprobe, anti-DIG-Rhodamine conjugated antibody (1:200, Roche) was used. DAPI was used to mark the nucleus. Samples were mounted in DABCO and observed under LSM 780 laser scanning confocal microscope Zeiss (Carl Zeiss). All the images were further processed using Adobe Photoshop 7.0.

\section{Statistical analysis}

In our study experiments were conducted in triplicate, analysis was done using PRISM 5 as guided (GraphPad, San Diego, CA) and results were given as the mean \pm standard deviation (S.D.). The statistical analysis was performed using one-way ANOVA. The significant level was set as $p$-values below 0.05 .

\section{Acknowledgements}

The authors acknowledge the Bloomington Drosophila Stock Centre and Vienna Drosophila Resource Centre. We thank Prof. James Castelli-Gair Hombría and Prof. Erika Bach for kindly providing us fly stocks and antibody.
We thank Prof. S.C. Lakhotia for providing hsrw construct for RNA-FISH control experiments. We acknowledge the real-time facility at ISLS (Interdisciplinary Centre for Life Sciences) and Department of Molecular and Human Genetics. We also acknowledge confocal facility at ISLS and Department of Zoology, Banaras Hindu University. B.M., R.D., and P.P. were supported by a fellowship from the Council of Scientific and Industrial Research, Government of India. SERB-POWER Fellowship to MM from Science and Engineering Research Board, Department of Science and Technology, Government of India is acknowledged.

\section{Author contributions}

Conceptualization, B.M., and M.M.; Methodology, B.M. and P.P.; Investigation, B. M., A.M., S.S., and M.M.; Software and Formal Analysis, B.M., R.D., and M.M.; Writing Original Draft, B.M. and M.M.; Supervision, M.M.

\section{Conflict of interest}

The authors declare no competing interests.

\section{Publisher's note}

Springer Nature remains neutral with regard to jurisdictional claims in published maps and institutional affiliations.

Supplementary information The online version contains supplementary material available at https://doi.org/10.1038/s41419-021-03649-0.

Received: 14 December 2020 Revised: 17 March 2021 Accepted: 18 March 2021

Published online: 06 April 2021

\section{References}

1. Keene, J. D. RNA regulons: coordination of post-transcriptional events. Nat. Rev. Genet. 8, 533-543 (2007).

2. Moore, M. J. From birth to death: the complex lives of eukaryotic mRNAs Science 309, 1514-1518 (2005).

3. Abdelhaleem, M. Do human RNA helicases have a role in cancer? Biochim. Biophys. Acta 1704, 37-46 (2004).

4. Fuller-Pace, F. V. DEAD box RNA helicase functions in cancer. RNA Biol. 10, 121-132 (2013).

5. Anthony, K. \& Gallo, J.-M. Aberrant RNA processing events in neurological disorders. Brain Res. 1338, 67-77 (2010).

6. Shamseldin, H. E. et al. Mutations in DDX59 implicate RNA helicase in the pathogenesis of orofaciodigital syndrome. Am. J. Hum. Genet. 93, 555-560 (2013).

7. Paine, I. et al. Paralog studies augment gene discovery: DDX and DHX Genes. Am. J. Hum. Genet. 105, 302-316 (2019).

8. Balak, C. et al. Rare de novo missense variants in RNA helicase DDX6 cause intellectual disability and dysmorphic features and lead to P-Body defects and RNA dysregulation. Am. J. Hum. Genet. 105, 509-525 (2019).

9. Lessel, D. et al. De novo missense mutations in DHX30 impair global translation and cause a neurodevelopmental disorder. Am. J. Hum. Genet. 101, 716-724 (2017)

10. Surabhi, S. et al. Regulation of notch signaling by an evolutionary conserved DEAD box RNA helicase, maheshvara in Drosophila melanogaster. Genetics 201, 1071-1085 (2015).

11. Salpietro, V. et al. A loss-of-function homozygous mutation in DDX59 implicates a conserved DEAD-box RNA helicase in nervous system development and function. Hum. Mutat. 39, 187-192 (2018).

12. Honig, L. S. \& Rosenberg, R. N. Apoptosis and neurologic disease. Am. J. Med. 108, 317-330 (2000)

13. Cavallaro, S. Cracking the code of neuronal apoptosis and survival. Cell Death Dis. 6, e1963 (2015).

14. Wang, Y. H. \& Huang, M. L. Organogenesis and tumorigenesis: insight from the JAK/STAT pathway in the Drosophila eye. Dev. Dyn. 239, 2522-2533 (2010).

15. Zeidler, M. P. \& Bausek, N. The Drosophila Jak-Stat pathway. JAKSTAT 2, e25352 (2013). 
16. Kisseleva, T., Bhattacharya, S., Braunstein, J. \& Schindler, C. Signaling through the JAK/STAT pathway, recent advances and future challenges. Gene $\mathbf{2 8 5}$ $1-24$ (2002).

17. Harrison, D. A., McCoon, P. E., Binari, R., Gilman, M. \& Perrimon, N. Drosophila unpaired encodes a secreted protein that activates the JAK signaling pathway. Genes Dev. 12, 3252-3263 (1998).

18. Mukherjee, T., Schäfer, U. \& Zeidler, M. P. Identification of Drosophila genes modulating Janus kinase/signal transducer and activator of transcription signal transduction. Genetics 172, 1683-1697 (2006).

19. Tsai, Y. C. \& Sun, Y. H. Long-range effect of upd, a ligand for Jak/STAT pathway, on cell cycle in Drosophila eye development. Genesis 39, 141-153 (2004).

20. Bach, E. A. et al. GFP reporters detect the activation of the Drosophila JAK STAT pathway in vivo. Gene Expr. Patterns 7, 323-331 (2007).

21. Borensztejn, A., Boissoneau, E., Fernandez, G., Agnès, F. \& Pret, A.-M. JAK/STAT autocontrol of ligand-producing cell number through apoptosis. Development 140, 195-204 (2013).

22. Hanratty, W. P. \& Dearolf, C. R. The Drosophila Tumorous lethal hematopoietic oncogene is a dominant mutation in the hopscotch locus. Mol. Gen. Genet. 238, 33-37 (1993).

23. Ma, C., Zhou, Y., Beachy, P. A. \& Moses, K. The segment polarity gene hedgehog is required for progression of the morphogenetic furrow in the developing Drosophila eye. Cell 75, 927-938 (1993).

24. Treisman, J. E. \& Rubin, G. M. wingless inhibits morphogenetic furrow movement in the Drosophila eye disc. Development 121, 3519-3527 (1995).

25. Heslip, T. R., Theisen, H., Walker, H. \& Marsh, J. L. Shaggy and dishevelled exert opposite effects on Wingless and Decapentaplegic expression and on positional identity in imaginal discs. Development 124, 1069-1078 (1997).

26. Greenwood, S. \& Struhl, G. Progression of the morphogenetic furrow in the Drosophila eye: the roles of Hedgehog, Decapentaplegic and the Raf pathway. Development 126, 5795-5808 (1999).

27. Baonza, A. \& Freeman, M. Notch signalling and the initiation of neural development in the Drosophila eye. Development 128, 3889-3898 (2001).

28. Fu, W. \& Baker, N. E. Deciphering synergistic and redundant roles of Hedgehog, Decapentaplegic and Delta that drive the wave of differentiation in Drosophila eye development. Development 130, 5229-5239 (2003).

29. Nicolas, C. S. et al. The role of JAK-STAT signaling within the CNS. JAKSTAT 2, e22925 (2013)

30. Colodner, K. J. \& Feany, M. B. Glial fibrillary tangles and JAK/STAT-mediated glial and neuronal cell death in a Drosophila model of glial tauopathy. $J$. Neurosci. 30, 16102-16113 (2010).

31. Qin, H. et al. Inhibition of the JAK/STAT pathway protects against a-synucleininduced neuroinflammation and dopaminergic neurodegeneration. J. Neurosci. 36, 5144-5159 (2016).
32. Hoi, C. S., Xiong, W. \& Rebay, I. Retinal axon guidance requires integration of Eya and the Jak/Stat pathway into phosphotyrosine-based signaling circuitries in Drosophila. Genetics 203, 1283-1295 (2016).

33. Bach, E. A., Vincent, S., Zeidler, M. P. \& Perrimon, N. A sensitized genetic screen to identify novel regulators and components of the Drosophila janus kinase/ signal transducer and activator of transcription pathway. Genetics 165, 1149-1166 (2003)

34. Harrison, D. A., Binari, R., Nahreini, T. S., Gilman, M. \& Perrimon, N. Activation of a Drosophila Janus kinase (JAK) causes hematopoietic neoplasia and developmental defects. EMBO J. 14, 2857-2865 (1995).

35. Brown, S., Hu, N. \& Hombría, J. C.-G. Identification of the first invertebrate interleukin JAKVSTAT receptor, the Drosophila gene domeless. Curr. Biol. 11, 1700-1705 (2001).

36. Wang, Y.-H. \& Huang, M.-L. Reduction of Lobe leads to TORC1 hypoactivation that induces ectopic Jak/STAT signaling to impair Drosophila eye development. Mech. Dev. 126, 781-790 (2009).

37. Tripathi, B. K., Surabhi, S., Bhaskar, P. K., Mukherjee, A. \& Mutsuddi, M. The RNA binding $\mathrm{KH}$ domain of Spoonbill depletes pathogenic non-coding spinocerebellar ataxia 8 transcripts and suppresses neurodegeneration in Drosophila. Biochim. Biophys. Acta 1862, 1732-1741 (2016).

38. Maurya, B., Surabhi, S., Pandey, P., Mukherjee, A. \& Mutsuddi, M. in Insights Into Human Neurodegeneration: Lessons Learnt From Drosophila 373-403 (Springer, 2019).

39. Dean, J. L. et al. The $3^{\prime}$ untranslated region of tumor necrosis factor alpha mRNA is a target of the mRNA-stabilizing factor HuR. Mol. Cell Biol. 21, 721-730 (2001).

40. Sawaoka, H., Dixon, D. A., Oates, J. A. \& Boutaud, O. Tristetraprolin binds to the 3'-untranslated region of cyclooxygenase-2 mRNA a polyadenylation variant in a cancer cell line lacks the binding site. J. Biol. Chem. 278, 13928-13935 (2003).

41. Katsanou, V. et al. HuR as a negative posttranscriptional modulator in inflammation. Mol. Cell 19, 777-789 (2005).

42. Winzen, R. et al. Functional analysis of KSRP interaction with the AU-rich element of interleukin-8 and identification of inflammatory mRNA targets. Mol. Cell Biol. 27, 8388-8400 (2007).

43. Duggimpudi, S. et al. Transcriptome-wide analysis uncovers the targets of the RNA-binding protein MSI2 and effects of MSI2's RNA-binding activity on IL-6 signaling. J. Biol. Chem. 293, 15359-15369 (2018).

44. Buchon, N., Broderick, N. A., Poidevin, M., Pradervand, S. \& Lemaitre, B. Drosophila intestinal response to bacterial infection: activation of host defense and stem cell proliferation. Cell Host Microbe 5, 200-211 (2009).

45. Schmittgen, T. D. \& Livak, K. J. Analyzing real-time PCR data by the comparative $C_{T}$ method. Nat. Protoc. 3, 1101-1108 (2008). 